\title{
5
}

\section{Electrical Heart Instability Evaluation in Conditions of Diastolic Heart Failure Suffered by Coronary Heart Disease Patients}

\author{
E.P. Tatarchenko, N.V. Pozdnyakova, O.E. Morozova and E.A. Petrushin \\ Penza Extension Course Institute for Medical Practitioners \\ Russia
}

\section{Introduction}

In 1965 WHO experts singed out coronary heart disease (CHD) in a separate group. It was dictated by the growing epidemic incidence rate and high mortality from disease complications. Besides, it was urgent to take measures to treat the disease. However, the end of the XX century and the beginning of the XXI century have not brought any significant changes. The heart and vessel diseases complicated by atherosclerosis are still one of the main problems in most countries. The main reasons are high incidence of disease, stabile director disability and mortality among employable population. Each year, cardiovascular diseases cause 4.3 million deaths in Europe in general, and over 2 million in the EU, accounting for 48 and $42 \%$ of the total number of deaths respectively. Mortality from coronary heart disease among of men in the age of 65 is 3 times higher than among women. In older age mortality rate is equalized, and after 80 years of age it is 2 times higher among women.

In its essence, all the pathophysiological manifestations of coronary heart disease are caused by an imbalance between myocardial oxygen demand and oxygen delivery.

CHD may start abruptly with myocardial infarction (MI) or sudden cardiac death (SCD), but almost $50 \%$ of patients suffer immediately a chronic form of coronary heart disease. It is called exertional angina [2, 3]. In absolute figures it means approximately 30,000 - 40,000 patients with angina per 1 million population [4]. According to some reports men suffering angina live on average eight years less than those who do not have this pathology [5].

According to epidemiological studies, CHD patients suffer blood circulation blocking in most cases; it leads to approximately $90 \%$ sudden deaths. About half of patients with diagnosticated CHD die suddenly without a preceding pain syndrome.

Meanwhile, M.R.Cowie and others [2002] argue, the main risk factor for sudden death is left ventricular dysfunction. The prognosis of chronic heart failure (CHF) is still extremely serious, regardless of its etiology, but you should agree with J.N. Cohn and others [1999] who wrote that "...coronary disease may be an independent predictor of poor prognosis for patients suffering heart failure". Traditionally, CHF was considered to have connection with systolic dysfunction, however, in recent years the main subject of research of clinicians and physiologists is mechanisms of myocardium diastolic dysfunction (DD) development and 
its role in the onset of heart failure. Nowadays heart failure is considered as a syndrome which develops from various pathological heart changes, neuroendocrinal regulation disorders and represents a complex of circulatory reactions because of systolic or diastolic cardiac dysfunction.

About $50 \%$ of patients with chronic heart failure die within 5 years after the onset of clinical symptoms despite the use of combination therapy. According to the Framingham study, $75 \%$ of men with $\mathrm{CHF}$ and $62 \%$ of women die within 5 years after establishing diagnosis. Only half of patients with CHF die from heart failure which is refractory to therapy. The second half of patients with heart failure dies suddenly because of ventricular tachyarrhythmia. Oxymortia is the main death mechanism (in $30-80 \%$ of cases) among patients with CHF of II-III of functional class [8]. These facts allowed us to formulate an assumption that heart failure is the most the arrhythmogenic factor in cardiology and the most important sign of sudden death risk $[9,10]$.

Thus, the prediction and solution of sudden cardiac death (SCD) problem is only possible with the full study of the structural abnormalities and functional diseases which cause lifethreatening arrhythmias acoording to modern model. Pathological changes of myocardium go with various dysfunctions of electrical heart activity. They are prognostically unfavorable in terms of the occurrence of fatally dangerous rhythm disturbances. The validity of the assumption is based on the fact that electrophysiological alternation of cells and cell membranes boosts the development of electrical heart instability (EHI) after the cases of transient coronary heart disease and myocardial infarction having developed in areas of myocardial dysfunction.

Though this problem is intensively investigated, the search of pathogenic mechanisms causing electrophysiological properties disorder and associated with pathological electrocardiographic and electrophysiological phenomena going with structural remodeling of myocardium in CHD is still important.

The research objective was to study the indicators characterizing electrical instability of myocardium suffered by CHD patients who have diastolic heart failure.

According to protocol adopted by local Ethical Committee a number of patients who had suffered myocardial infarction more than a year ago took part in the research. They had a stabile clinical course of coronary heart disease and clinical implications of chronic cardiac failure syndrome with left ventricle ejection fraction of $45 \%$ during the previous month. Each patient signed an agreement to take part in our research as a volunteer. We observed the group of 128 patients ( 36 women and 92 men). The average age of patients was $57.3 \pm 5.6$ years.

The elimination criteria were coronary revascularization or cerebral stroke during the last 6 months, symptoms of VI class cardiac failure according to NYHA classification, clinically significant cardiac defects and lung diseases, dysfunctions of liver and nephros, atrial fibrillation.

Besides standard clinical research, we did the whole complex of work including electrocardiography in 12 derivations, echocardiography, Holter monitoring, registration of average signaling electrocardiography with identification of ventricular late potential, analysis of variability of cardiac rhythm, evaluation of ventricle repolarization-interval dispersion $\mathrm{Q}-\mathrm{T}\left(\mathrm{QT}_{\mathrm{d}}\right)$, resolved interval $\mathrm{Q}-\mathrm{T}(\mathrm{QT} \mathrm{C})$. 
We evaluated left ventricular geometry and left atrium (end-diastolic size - EDD, size - ESD; end-systolic volume - EDV, end-systolic volume - ESV; left ventricular mass index - LVMI, relative thickness of the left ventricular wall; left atrium volume change index); left ventricular systolic function (ejection fraction - EF, \%; stroke index - SI, $\mathrm{ml} / \mathrm{m}^{2}$; systolic shortening`s fraction of the front-back size aortic ventricle - $\Delta \mathrm{S}, \%$ ); myocardium regional contractility (diacrisis of zone with regional contractility dysfunction). We used ultrasound cordis investigation with Doppler spectral echocardiography mode and color Doppler mapping.

Analyzing left ventricular diastolic function we studied indeces of transmitral diastolic flow in incipient and delayed diastole (E, A, m/s), their ratio (E/A), time of flow delay(DT, ms) and flow acceleration during the phase of rapid inflow(AT, ms), is ovolumic relaxation time (IVRT, ms) and duration of diastole (ET, ms) - Figure 1.

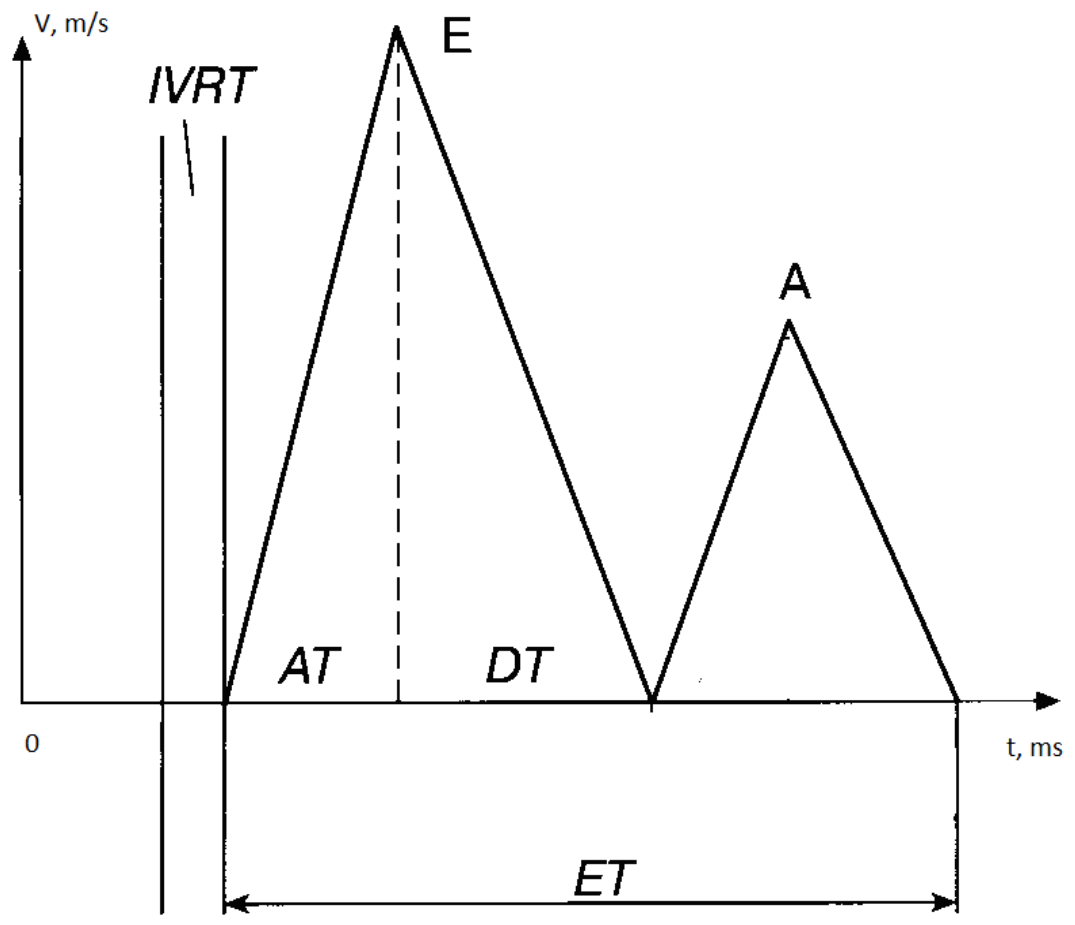

Fig. 1. Estimation of trancmitral diastolic flow

We also evaluated blood flow at the mouth of the pulmonary veins (D, cm / s; Ar, cm / s; Adur / Ar) - Figure 2.

We used tissue Doppler sonography to detect signs of inflow disorder, loss of elasticity, the increase of the left ventricular rigidity. We defined the maximum speed of mitral annulus: peak systolic speed - S '(cm / s) peak speed of incipient diastolic relaxation - E' (cm / s) peak speed in atrial systole phase - A ' $(\mathrm{cm} / \mathrm{s})$, the ratio of maximum speed of incipient LV inflow (E) to the maximum speed of the fibrous ring kinesis in incipient diastole (E') - Figure 3. 


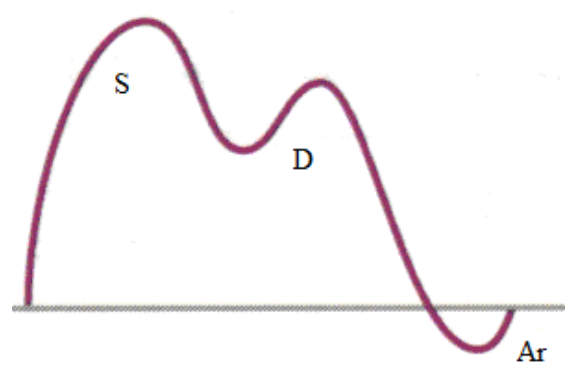

Fig. 2. Estimation of bloodstream at the mouth of the pulmonary veins

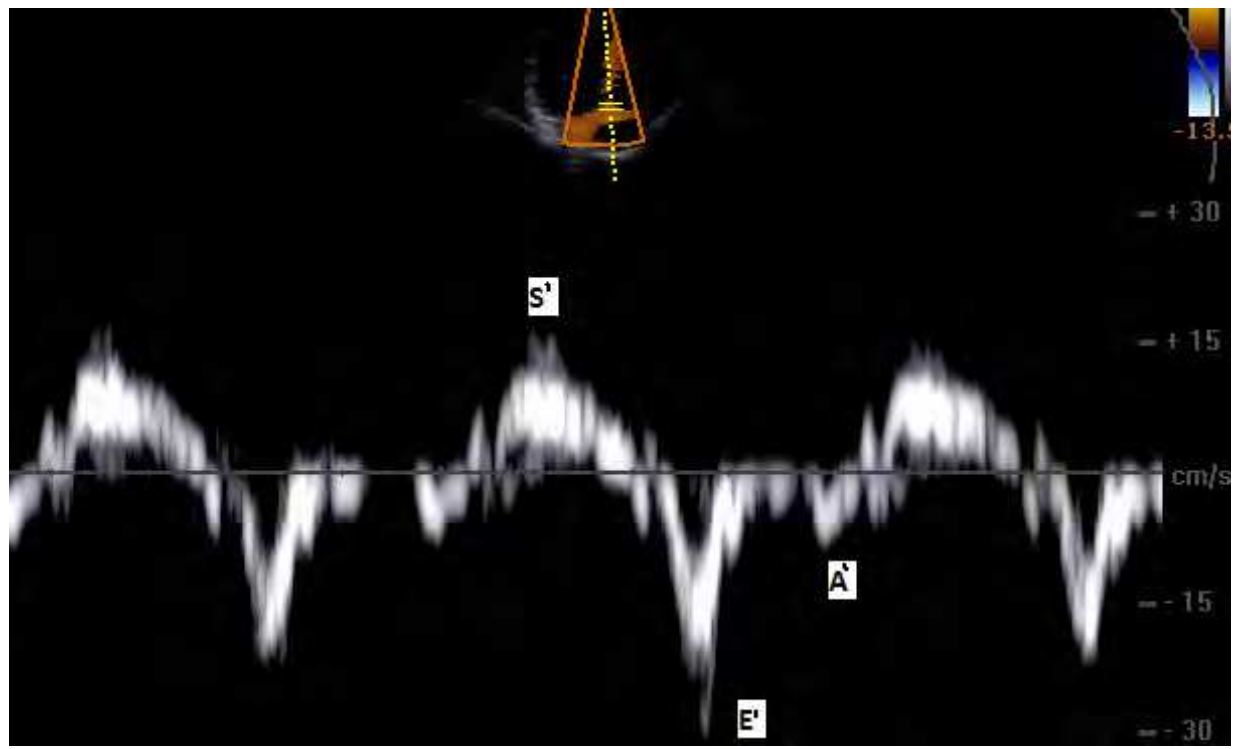

$\mathrm{S}^{\prime}(\mathrm{cm} / \mathrm{s})$ - peak diastolic speed; $\mathrm{E}^{\prime}(\mathrm{cm} / \mathrm{s})$ - peak speed of incipient diastolic relaxation; $\mathrm{A}^{\prime}(\mathrm{cm} / \mathrm{s})$ peak speed in atrial systole phase.

Fig. 3. Fibrous ring`s movement of the mitral valve in pulse-wave mode histic doppler

Using Holter monitoring ECG we took into account the nature of rhythm disturbance and asequence, we estimated the quantity of pain assessed and painless ischemic episodes, the daily duration of ischemia, the maximum depth of segment ST depression.

Time-line analysis of ventricular late potential was performed by method of M. Simson [1981]. We calculated numerical quantitative values of three indicators: the duration of the filtered complex QRS (HF QRS-Dauer), the rms amplitude of the last $40 \mathrm{~ms}$ of the complex QRS (RMS 40), the duration of the low amplitude signals at the end of the filtered complex QRS (LAH Fd). We took HF QRS-Dauer which is more than 114 ms, RMS 40 which is less than $25 \mathrm{mV}$, LAH Fd which is more than $38 \mathrm{~ms}$ as pathological parameters of the signal averaged ECG - Figure 4. 


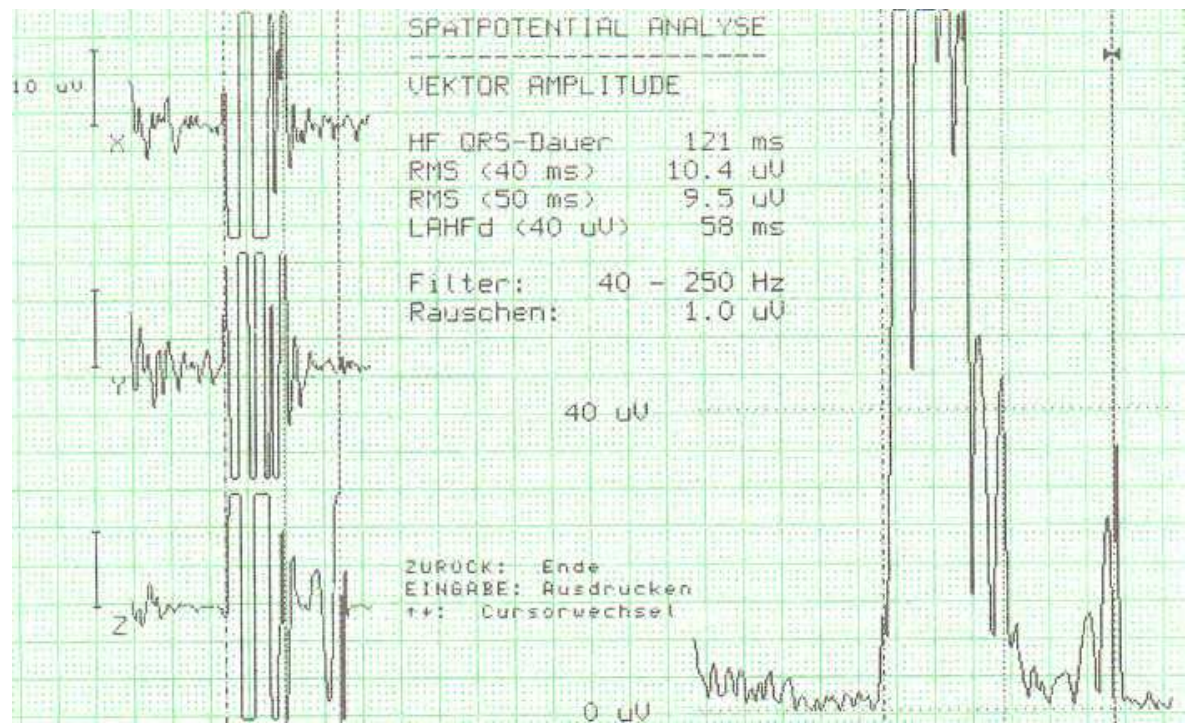

Fig. 4. Analysis of the signal-averaged ECG with elimination late potentials of the ventricles

Performing heart rate variability analysis we evaluated rMSSD (mean square difference between the duration of contiguous sinus intervals RR, BB 50 (proportion of contiguous sinus intervals RR which differ more than $50 \mathrm{~ms}$, \%), SDNN (standard derivation of average duration of sinus intervals RR, ms).

Performing spectral analysis of heart rate variability analysis we estimated frequency ranges: high frequency $(\mathrm{HF})$, low frequency $(\mathrm{LF})$, the ratio of sympathetic and parasympathetic influences on heart rate variability $(\mathrm{LF} / \mathrm{HF})$.

We examined the following heart rate turbulence indicants:

- turbulence onset $(\mathrm{TO}, \%)$. We estimated the value-of sinus rhythm acceleration after ventricular arrhythmia;

- turbulence slope (TS, ms / RR). We studied the intensity of the sinus rhythm deceleration, following its quickening.

In assessing ventricular repolarization we calculated corrected interval Q-T (QTs) using Bazeta. The dispersion of the interval Q-T (QTd) was defined as the difference between the maximal and minimal value of Q-T interval in different leads of standard ECG.

T-test of Student, entry criteria applied by static data handing. Gotten results have been presented in the form of an average arithmetical significance \pm is standart devition. Differences considered reliable at $\mathrm{p}<0,05$.

In case of CHD left ventricle diastole indices and diastole functions undergo complex changes which are connected with both worsening of diastolic disorders and the development of hemodynamic adaptive responses acting through an increase in pressure in the left atrium and/or left ventricular end diastolic pressure and leading to the formation of various types of diastolic dysfunction. 
We divided our patients into 3 groups according to the type of diastolic dysfunction of left ventricle: the first group $(n=36)$ included patients with abnormal relaxation of left ventricle, the second group $(n=28)$ consisted of patients with pseudonormal type of diastolic dysfunction and the third group $(n=22)$ was formed from the patients who suffered restrictive type of diastolic dysfunction of left ventricle.

The average duration of disease was $6.9 \pm 3.9$ years ( 3 to 12 years). Medical history and electrocardiographic criteria indicated macrofocal myocardial infarction (with $Q$ tooth) suffered by 68 patients (53.1\%).9 out of 68 patients had a chronic aneurysm of the anterior wall according to electrocardiographic and echocardiographic features. Fine-focal MI (without $\mathrm{Q}$ tooth) is marked in 60 cases according to medical documentation (hospital records, discharge summary). 82 patients $(64 \%)$ had coronary heart disease accompanied by arterial hypertension (AH). 92 patients (72\%) had burdened familial history. Lipid exchange violations were indicated in 112 cases $(87.5 \%)$.

Figure 1 shows the gradation of patients with different variants of the left ventricle diastolic dysfunction according to functional classes of chronic heart failure (CHF). 42 patients had signs of I FC CHF, 51 patients had II FC and 35 patients had III FC.

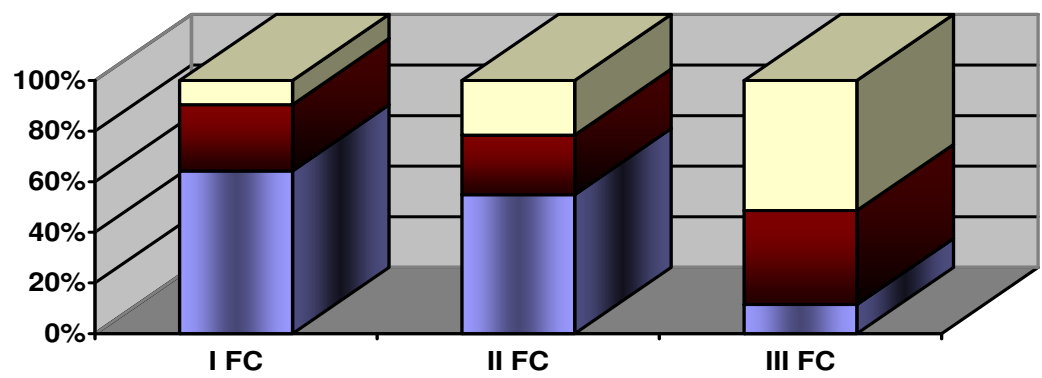

\section{$\square$ diastolic dysfunction of abnormal relaxation type $\square$ pseudonormal type of diastc $\square$ restrictive type of diastolic dysfunction}

Fig. 5. The gradation of patients suffering coronary heart disease with left ventricle diastolic dysfunction according to functional classes of chronic heart failure (CHF).

In cases of heart failure clinical manifestations we have received the relation of $r=0,620$ ( $p$ $<0.01$ ) of the frequency of CHF FC to the type of diastolic dysfunction. When FC increases the disturbances of LV DF become more pronounced: restrictive type of LV DD was detected in III FC (51\% observations); in I FC it was observed on $11.4 \%$; patients had the type of abnormal relaxation in $9.5 \%$ and $64.3 \%$ respectively, pseudonormal type was detected in $21.6 \%$ and $55 \%$ of cases.

The analysis of the structural and geometric parameters of left heart chambers (Table 1) showed that group III had an increase of the dilation of the cavities of the left ventricle and left atrium, increased left ventricle myocardial mass, reduction in contractile function 
(ejection fraction of III group was more than $45 \%$ but less than $50 \%$, in group I it was $50 \%$ ) in comparison with group I.

\begin{tabular}{|c|c|c|c|}
\hline Indicators & I group & II group & III group \\
\hline Number of patients, $n$ & 59 & 36 & 33 \\
\hline $\mathrm{E}, \mathrm{m} / \mathrm{s}$ & $0.65 \pm 0.06^{*}$ & $0.81 \pm 0.03$ & $1.07 \pm 0.05^{* *}$ \\
\hline $\mathrm{A}, \mathrm{m} / \mathrm{s}$ & $0.93 \pm 0.09 *$ & $0.65 \pm 0.02$ & $0.36 \pm 0.03^{*}$ \\
\hline $\mathrm{E} / \mathrm{A}$ & $0.82 \pm 0.05^{*}$ & $1.25 \pm 0.02$ & $3.15 \pm 0.08^{* *}$ \\
\hline $\mathrm{AT}, \mathrm{ms}$ & $99.47 \pm 2.1$ & $94.1 \pm 3.2$ & $97.3 \pm 3.3$ \\
\hline $\mathrm{DT}, \mathrm{ms}$ & $267.6 \pm 11.4^{*}$ & $169.6 \pm 7.9$ & $143.5 \pm 3.2^{* *}$ \\
\hline IVRT, ms & $116.8 \pm 7.4^{*}$ & $88.3 \pm 2.3$ & $58.9 \pm 4.8^{* *}$ \\
\hline $\mathrm{Ar}, \mathrm{cm} / \mathrm{s}$ & $24.1 \pm 2.2^{\circ}$ & $36.6 \pm 1.3$ & $55.3 \pm 1.6$ \\
\hline $\mathrm{SI}, \mathrm{ml} / \mathrm{m}^{2}$ & $40.3 \pm 2.8$ & $41.2 \pm 1.4$ & $41.7 \pm 2.6$ \\
\hline $\mathrm{EDVI}, \mathrm{ml} / \mathrm{m}^{2}$ & $82.1 \pm 5.3^{*}$ & $85.8 \pm 4.58$ & $100.8 \pm 6.7$ \\
\hline ESVI, $\mathrm{ml} / \mathrm{m}^{2}$ & $37.85 \pm 2.1^{*}$ & $39.2 \pm 1.1$ & $41.61 \pm 2.5$ \\
\hline LVEF, \% & $55.93 \pm 2.7^{*}$ & $53.1 \pm 2.17$ & $49.6 \pm 2.06$ \\
\hline ESVILA, ml/m² & $39.5 \pm 2.3^{*}$ & $44.37 \pm 3.2$ & $50.43 \pm 3.61$ \\
\hline ICVLA, \% & $37.65 \pm 1.5$ * & $34.1 \pm 2.1$ & $24.9 \pm 2.7$ \\
\hline LVMI, g/m² & $126.35 \pm 9.7^{*}$ & $132.1 \pm 6.3$ & $148.2 \pm 11.1$ \\
\hline left ventricular RT, $\mathrm{cm}$ & $0.44 \pm 0.06^{*}$ & $0.45 \pm 0.05$ & $0.50 \pm 0.02$ \\
\hline
\end{tabular}

SI - stroke index, EDVI - end-diastolic volume index, ESVI - end systolic volume index, LVEF - left ventricular ejection fraction, ESVILA - end systolic volume index of left atrium, the ICVLA - an indicator of changes in the volume of the left atrium, LVMI - left ventricular mass index, left ventricular RT is the relative thickness of the left ventricular wall, ${ }^{*}-\mathrm{p}<0.05$ - reliability of differences between patients with DD of abnormal relaxation type and patients with pseudonormal type of DD, ${ }^{* *} \mathrm{p}<0.05$ - reliability of differences between the group of patients with restrictive type of DD and the groups with pseudonormal type of DD and abnormal relaxation, ${ }^{\circ}-p<0.05$ - reliability of the indicator difference between group with DD of abnormal relaxation type and groups with DD of pseudonormal and restrictive type.

Table 1. Echocardiographic indicators of patients with coronary heart disease

We believe that one of the determinants of heart failure is diastolic dysfunction of the heart, which can be viewed both as the initial stage and as a pathophysiological link in the "vicious circle" of circulatory inefficiency, which affects negatively on the prognosis of patients with coronary heart disease [11].

The violation of left ventricular diastolic properties is essential, along with other structural and functional changes (dilation and hypertrophy), which form the concept of "remodeling". Patients had eccentric hypertrophy $(43.75 \%$ of cases) and concentric hypertrophy of LV (37.5\% of cases) as predominant types of LV remodeling after MI. 
We established the correlation between the severity of LV DD and exponent change in the volume of the left atrium $-\mathrm{r}=-0.43(\mathrm{p}=0.032)$, with left ventricular ejection fraction $-\mathrm{r}=-$ $0.48(p<0.04)$, with an index of end diastolic volume $-r=0,51(p=0.02)$, with left ventricular mass index $-r=0.45(p=0.034)$.

We associate the appearance and progression of LV DD with violation of diastolic relaxation on the back of acute or long-lasting ischemia with increased stiffness of the myocardium, including the location of postinfarction scar. We spotted the following law: a degree of LV DF disorder was growing in patients having macrofocal myocardial infarction versus patients having fine-focal myocardial infarction, respectively, it was $30.5 \%$ with abnormal relaxation type and $69.5 \%(p=0.047), 58.3 \%$ and $41.7 \%(p=0.05)$ with pseudonormal, $87.9 \%$ and $12.1 \%(p=0.0476)$ with restrictive type. We believe that patients with previous Qinfarction had long-lasting deterioration of LV function during myocardial hibernating, which is characterized by a chronic reduction in cardiomyocyte contractility, while their viability is maintained. In these conditions viable myocardium which is far from infarction zona undergoes diastolic myocardial stress.

We detected the dependence of manifestation degree of diastolic dysfunction with an index of contractility WMSI $-\mathrm{r}=0.91(\mathrm{p}=0.01)$ among patients with a history of MI. We pointed the positive correlation of restrictive type of LV DD with Q- myocardial infarction $r=0.638$ ( $p<0.001$ ). In group III (restrictive type) Q-infarction occurred in $87.9 \%$ of cases, which is significantly lower in comparison with group I $-30.5 \%, \mathrm{p}<0.01$.

In conditions of limited blood flow the possibility of injury and death of cardiomyocytes because of their necrotization and apoptosis [12] is the main unfavorable result of myocardial hypoxia in case of coronary heart disease. However, death of cardiomyocytes is accompanied by compensatory hypertrophy of survived myocardial cell and the development of connective tissue [13]. There is an opinion that left ventricle hypertrophy may lead to myocardial dysfunction. It is an individual risk factor for high cardiovascular case rate, including ischemia myocardial infarction, heart failure, ventricular arrhythmias and sudden death.

We tend to assume that the process of cardiac remodeling affects all myocardium components. Changes occur at the level of cardiac myocytes and in the extracellular matrix. Typically, ischemic myocardial injuries develop leading to the coagulation and apoptosis. In this case, as approved by the J.Soto, G.Beller [2001], responsive genes are activated immediately, especially c-fos, and the program of "programmed death", apoptosis, starts to run. In the peripheral areas ischemic injury usually results in colliquative necrosis with cell edema and myocytolysis. It is especially true for reperfusion injury. The formation of connective tissue (fibrosis) may have redundant diffuse or interstitial nature in addition to substitution (focal), with interstitial spread perivascular.

Different biomechanical properties of myocardium in chronic ischemic zone and so-called intact myocardium generate special effects according to the type of stress concentration on their border. The type of stress concentration is connected with further progression of degenerative and sclerotic changes, significant changes in the shape and size of remodeled heart chambers. Further LV remodeling with the expansion of the cavity in postinfarction period is caused by involving of myocardium in the "transition zone" in the process of remodeling. The data obtained from pathophysiological studies suggest that patients suffering coronary heart disease have clinical picture determined by not only the 
heterogeneity of causes, but also the diversity of adaptive and disadaptive changes in metabolism and contractile condition of cardiomyocytes [16]. The most significant adaptive myocardium reactions in response to myocardial ischemia include the "new ischemic syndromes": hibernate, stupor.

The defining condition for the occurrence of lethal arrhythmias is considered to be structural disease of heart, which becomes electrically unstable under the influence of various functional factors. Ischemia and necrosis, hypertrophy and dilatation of the ventricles, the inflammation and swelling of the myocardial tissue can act as the structural changes that determine the development of ventricular tachycardia. According to the results of many studies [17, 18, 19] these changes constitute anatomical substrate for appearance of malignant arrhythmias with the participation of the various triggering and modulating factors.

Ventricular late potential with a stable disease course was recorded among 35 patients (27.3\%) with a history of MI (Table 2). We believe that structural cardiomyocyte changes, programmed apoptosis and secondary hypertrophy, increased diastolic stiffness and active myocardium relaxation violation, reactive changes of connective tissue skeleton of the myocardium, decrease in diastolic filling and remodeling with segmental structure violation, i.e. all these processes, lead to the formation of electrical myocardium heterogeneity and cause greater frequency of recording of late potentials among CHD patients in conditions of progression of LV DD: with abnormal relaxation (18.6\%), with pseudonormal type $(27.8 \%)$, and restrictive type of $\operatorname{LV}$ DD $(42.4 \%, \mathrm{p}<0.05)$.

\begin{tabular}{|c|c|c|c|}
\hline \multirow{2}{*}{ Indicator } & \multicolumn{3}{|c|}{ Variations of diastolic dysfunction } \\
\cline { 2 - 4 } & Type I & Type II & Type III \\
\hline \hline The number of patients, $\mathrm{n}$ & 59 & 36 & 33 \\
\hline $\begin{array}{c}\text { The number of patients with } \\
\text { ventricle late potential, } \mathrm{n}\end{array}$ & 11 & 10 & 14 \\
\hline $\begin{array}{c}\text { The number of patients with } \\
\text { ventricular arrhythmia, } \mathrm{n}\end{array}$ & 50 & 35 & 33 \\
\hline \multicolumn{4}{|c|}{ analysis of ventricular extrasystole } \\
\hline I gradation, $\mathrm{n} / \%$ & $29 / 49.1 \%$ & $10 / 27.8 \%$ & $2 / 6.1 \%$ \\
\hline IIgradation, $\mathrm{n} / \%$ & $7 / 11.9 \%$ & $7 / 19.4 \%$ & $5 / 15.1 \%$ \\
\hline IIIgradation, $\mathrm{n} / \%$ & $10 / 16.9 \%$ & $13 / 36.1 \%$ & $12 / 36.4 \%$ \\
\hline IV-V gradations, $\mathrm{n} / \%$ & $4 / 6.8 \%$ & $5 / 13.9 \%$ & $14 / 42.4 \%$ \\
\hline \multicolumn{4}{|c|}{ analysis of Q-T interval } \\
\hline RRNN, $\mathrm{ms}^{2}$ & $810 \pm 64$ & $798 \pm 98$ & $753 \pm 132$ \\
\hline $\mathrm{QT}_{\mathrm{c}}, \mathrm{ms}$ & $405.5 \pm 5.2^{*}$ & $422.3 \pm 2.6^{* *}$ & $450.2 \pm 5.4$ \\
\hline $\mathrm{QT}_{\mathrm{d}}, \mathrm{ms}$ & $39.7 \pm 2.7^{*}$ & $49.6 \pm 3.3^{* *}$ & $64.2 \pm 3.1$ \\
\hline
\end{tabular}

I type- LV DD of abnormal relaxation type, II type - LV DD of pseudonormal type; III type - LV DD of restrictive type, VLP ventricular late potential, VA- ventricular arrhythmia, RRNN - the duration of sinus RR intervals; $n$ / \% - the absolute number of patients with given symptom/percentage of the number of patients of this group.

Table 2. The comparative analysis of ventricular arrhythmias, cardiac late potentials, QT intervals among patients with coronary heart disease 
Ventricular arrhythmias are considered to be one of the factors determining the poor prognosis and high mortality among CHD patients. Ventricular arrhythmias may be the cause of death among patients with heart failure symptoms even in conditions of adequate control of decompensation symptoms.

Cardiac arrhythmias were detected among 99.2\% of patients (Table 2), while supraventricular arrhythmias (SA) were detected among $7 \%$ of patients, ventricular arrhythmias were detected among $56.2 \%$ of patients, a combination of ventricular arrhythmias (VA) with various forms of supraventricular arrhythmias (SA) were indicated among $36 \%$ of patients. Complex forms of VA (ventricular extrasystole of grades IV-V) were found in 23 cases (18\%). Polymorphic ventricular extrasystole was detected among $35 \mathrm{CHD}$ patients with left ventricular diastolic dysfunction (27.3\%). In 41 cases (32\%) the revealed ventricular extrasystole was assigned to I gradation when the frequency of episodes did not exceed 220 per day.

Having analyzed ventricular arrhythmias we noted the following law: early, paired, volley ventricular extrasystoles were detected among patients with slow fragmented activity more frequently in comparison with patients without ventricular late potential, respectively, 19 $(54.3 \%)$ and $4(4.3 \%)$ with $\chi^{2}=7.4, \mathrm{p}<0.001$.

Ventricular late potential and ventricular arrhythmias were recorded more often with an increase in the degree of diastolic dysfunction and end-diastolic volume of left ventricle. When DD had a restrictive type, ventricular extrasystole was found in $100 \%$ of cases and ventricular extrasystole of IV-V grades was detected among 14 patients (42.4\%), 13 of them had a diagnosed slow fragmented activity at the end of the ventricular complex. In group I (with abnormal left ventricular relaxation) ventricular extrasystole was observed among $84.7 \%$ of patients and complex forms of ventricular extrasystole were detected among 4 patients with abnormal rates of Holter ECG $(6.8 \%)$.

The most common mechanism for tachyarrhythmias of high grades is the mechanism of reentry momentum. It means the presence of unilateral and delayed passage of the depolarization wave front because of the violation of inter-cell contacts in parallel-oriented fibers [20], proliferation heterogeneity and fragmentation of the depolarization wavefront, which is a marked by ventricular late potential. That's what we explain the significant prevalence of complex forms of ventricular extrasystole among patients with slow fragmented ventricular activity (54.3\%).

To study the effect of reversible ischemia on myocardial electrophysiological properties we analyzed daily myocardial ischemia and indicators of Holter ECG among patients with coronary heart disease having left ventricular DD. Correlation analysis revealed the dependence of the daily duration of ischemia on the duration of filtered set of HF QRS Dauer $(r=0.53, p=0.01)$, with duration of low-amplitude signals at the end of filtercentered complex QRS LAH Fd $(r=0.49 p=0.03)$, duration of painless myocardial ischemia with HF QRS Dauer $(\mathrm{r}=0.57, \mathrm{p}=0.01)$. A positive correlation was obtained by estimating the depth of segment depression in HF QRS-Dauer $(r=0.45, p=0.03)$, with LAH Fd $(r=$ $0.41, \mathrm{p}=0.05)$; number of painless ischemic episodes per day with HF QRS-Dauer $(\mathrm{r}=0.51$, $\mathrm{p}=0.04)$. 
The results confirm that the presence of slow fragmented activity focus, marked by ventricular late potential is connected with heterogeneous areas of myocardium in CHD patients: profound disturbances in the processes of relaxation and recovery of cardiomyocytes occur in both acute and chronic ischemia because of degenerative changes and apoptosis. But it should be noted that studying the complex mechanisms of late potentials formation and the functions and metabolism of cardiac muscle, we traditionally pay much attention to cardiomyocytes. However, we tend to assume that extracellular matrix plays a insignificant role in the genesis of fragmented ventricular activity. Fibrosis is an essential component of cardiac muscle remodeling; the development of reparative (replacement) fibrosis may not only violate cardiomyocytes supply but also impede the electrical contact between them [21] in case of ischemia and myocardial necrosis.

The results of our studies have shown that myocardial dysfunction in ischemic area leads to the inhomogeneity of repolarization processes in conditions of limited coronary blood flow. However, the increase of QT dispersion in patients with coronary heart disease is associated with the duration of daily myocardial ischemia (DMI). We received a positive relationship of DMI with $\mathrm{QT}_{\mathrm{d}}(\mathrm{r}=0.485, \mathrm{p}=0.05)$. When the DMI was more than 60 minutes the duration of QTc and $\mathrm{QT}_{\mathrm{d}}$ interval was $449.2 \pm 4.1 \mathrm{~ms}$ and $67.2 \pm 2.7 \mathrm{~ms}, \mathrm{p}$ $<0.05$, when the DMI was 30 to $60 \mathrm{~min}$ analogous figures corresponded to the values of $412.5 \pm 3,2 \mathrm{~ms}$ and $46.8 \pm 2,4 \mathrm{~ms}$.

While studying QTd we observed higher values in patients with ventricular arrhythmia of III-V grades $(67.5 \pm 4.2 \mathrm{~ms})$ in comparison with patients without ventricular arrhythmia (44.3 $\pm 2.5 \mathrm{~ms}), \mathrm{p}<0,05$. The analysis revealed a dependence of the variance of QT interval with gradation of ventricular extrasystole $(r=0.6432, p<0.01)$, with exponents of Holter ECG: LAH Fd ( $r=0.4831, \mathrm{p}<0.05)$ HF QRS-Dauer $(\mathrm{r}=0.5744, \mathrm{p}<0.05)$.

The proportion of patients with restrictive type of LV DD with QTc interval which was over $440 \mathrm{~ms}(69 \%)$, and QTd which was more than $50 \mathrm{~ms}(72 \%)$ was significantly higher in comparison with those values in group II, it was $30.6 \%$ and $33 \%(p<0.05)$ respectively, and in Group I it was $19 \%$ and $17 \%(\mathrm{p}<0.03)$ respectively.

The performance analysis of electrical and structural remodeling among patients havingcoronary heart disease with different variants of left ventricular DD allowed us to establish a clear pattern (Figure 6): ventricular late potential and ventricular arrhythmias of higher grades were detected more often with increasing degree of diastolic function disturbance. Inhomogeneity of repolarization processes was heightened with an increase in QT interval dispersion. In our view, the resulting dependence reflects inhomogeneity of repolarization processes and depolarization of the ventricles in chronic ischemia, the conditions for malignant arrhythmias in CHD patients having left ventricular DD were created. We assume that the electrical stability of myocardium and its global-sustaining capacity are integral characteristics that determine the functioning of the heart as a selfregulating system. The violation of bioelectrical activity of the myocardium inevitably leads to its mechanical failure.

The discussion of the pathogenesis of coronary heart disease was always connected with neurogenic component. The theory of "punctuated antagonism" of the interaction of two divisions of the autonomic nervous system (ANS) and their impact on the regulation of the 
pacemaker in the sinus node (SN) is complicated by the activity of all the structures of the peripheral segmental heart ANS. In conditions of limited coronary blood flow morphofunctional component is considered to be slowly-varied, and pathologically altered regulation of heart rate variability is one of the earliest and most obligate manifestations of ischemic process [22, 23]. Sympathetic distress is a critical drop in power of neurohumoral regulation with a shift in the sympathovagal balances to the sympathetic component. It is regarded as a predictor of sudden death which is independent from left ventricular ejection fraction [22].

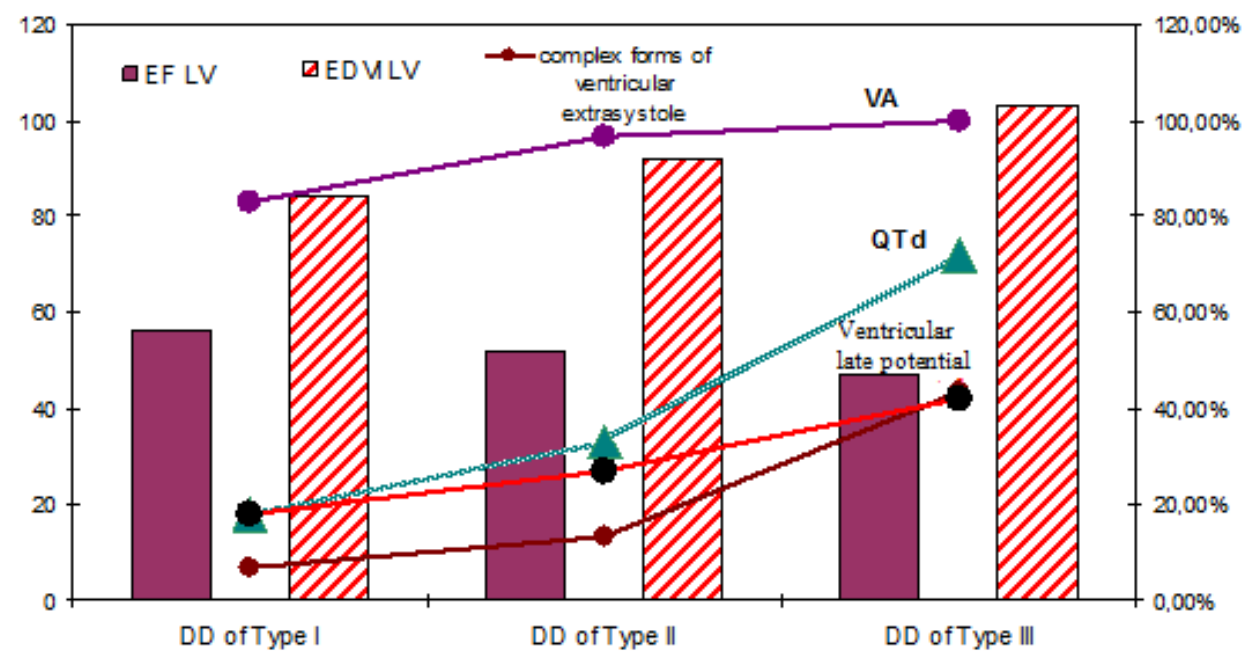

DD of Type I - diastolic dysfunction according to the type of abnormal relaxation, DD of Type IIdiastolic dysfunction of pseudonormal type, DD of Type III - diastolic dysfunction of restrictive type; EDVI LV - end-diastolic volume index of left ventricle, EF LV - ejection fraction left ventricular, QTd the dispersion of Q-T interval.

Fig. 6. The performance analysis of structural and geometrical and eletrical remodeling in CHD patients with left ventricle diastolic dysfunction.

Assessing the neurohumoral regulation of heart rate, we conducted the analysis of quantitative indicators of HRV and HRT (Table 3).

HRV indices in the group with impaired relaxation did not differ significantly from those in the group with pseudonormal type, once-reliable differences were obtained in comparison with III group of diastolic heart failure.

Rates BB50, SDNN index, rMSSD are drastically reduced in patients of group III. These values indicate a disorder of vagal protective effect on the value of "threshold of heart fibrillation". Disorders of neurohumoral regulation were diagnosed in conditions of the restrictive version of the DD LV, they pointed the increased sympathetic activity (SDNN 24.7 $\pm 3,2 \mathrm{~ms}, \mathrm{LF} / \mathrm{HF}-5.46 \pm 0,61$ conventional units, BB50 - 0\%).

During our study we pointed out the following pattern: autonomic imbalance with increased sympathetic activity grew with an increase in the degree of LV DD. The excess of 
the balance of ANS of sympathetic type LF / HF was observed in 39\% of patients in group I who had abnormal left ventricular relaxation, it was detected in $58.3 \%$ of patients in group II with pseudonormal type of DD LV and it was indicated in $94 \%$ cases in group III with restrictive type, $\mathrm{p}<0.05$.

And, if the HRV is a proven non-invasive method of quantitative analysis of the functioning of ANS in patients with coronary heart disease with preserved sinus rhythm, HRT is a fairly new noninvasive method of estimation the modulating autonomic influences on the sinus node in patients with ventricular arrhythmias, which resulted from the progressive fading of the carotid baroreflex [24]. Schematically, the formation of HRT can be represented by the following sequence: compensatory pause occurs after ventricular extrasystole, and, as a result, heart rate and blood pressure increase are caused by baroreflex, then blood pressure increase leads to the subsequent decrease in heart rate through baroreflex.

\begin{tabular}{|c|c|c|c|}
\hline Indicators & Group I & Group II & Group III \\
\hline \hline The number of patients, $\mathrm{n}$ & 59 & 36 & 33 \\
\hline \hline RRNN, ms & $810 \pm 64$ & $798 \pm 98$ & $753 \pm 132$ \\
\hline \hline \multicolumn{4}{|c|}{ analysis of heart rate variability } \\
\hline \hline SDNN, ms & $41.4 \pm 2.7$ & $36.6 \pm 2.3$ & $24.7 \pm 3.2^{*}$ \\
\hline \hline SDNN index, ms & $22.6 \pm 1.9$ & $19.3 \pm 1.3$ & $8.7 \pm 1.6^{*}$ \\
\hline \hline rMSSD, ms & $23.1 \pm 2.1$ & $21.1 \pm 1.4$ & $16 \pm 1.3^{*}$ \\
\hline \hline BB50, beats per minute & $10.3 \pm 3.2$ & $8.8 \pm 1.4$ & $3.4 \pm 1.2^{*}$ \\
\hline \hline LF/HF, conv. & $2.93 \pm 0.54$ & $3.51 \pm 0.68$ & $5.16 \pm 0.61^{*}$ \\
\hline \hline TO more 0\%, $\mathrm{n} / \%$ & $10 / 17$ & $9 / 25$ & $17 / 51.5$ \\
\hline \hline TS less 2,5Mc/RR, $\mathrm{m} \%$ & $9 / 15.2$ & $9 / 25$ & $16 / 48.5$ \\
\hline \hline
\end{tabular}

RRNN - the average duration of sinus RR intervals between contractions, SDNN-standard deviation from the mean duration of RR intervals between sinus contractions, BB50 - the number of adjacent sinus RR intervals, which differ by more than $50 \mathrm{~ms}$, SDNN index-average standard deviations, rMSSD square root of the sum of squared differences of successive sinus RR intervals, LF/HF - index of the balance of sympathetic and parasympathetic nervous systems; TO is more than $0 \%$; TS is less than 2.5 $\mathrm{ms} / \mathrm{RR}^{*}-\mathrm{p}<0,05$ - the difference is reliable when indicators of group III are compared with indicators of I and II groups.

Table 3. Quantitative indices of HRV in patients with coronary heart disease

HRT indicators reflect early acceleration of sinus rhythm (TO) and the subsequent decrease in heart rate (TS) after ventricular arrhythmia. Studies in recent years characterized HRT as effective method of predicting the outcomes of patients with coronary pathology, which is 
comparable with HRV, and in some cases it is superior to HRV in its diagnostic capabilities $[25,26]$.

During our study we reported the value of TO> $0 \%$ in 36 patients $(28 \%)$ from the group of 128 patients with previous MI history, we detected TS $<2.5 \mathrm{~ms} / \mathrm{RR}$ in 34 cases (26.6\%), and we observed the combination of pathological values of TO and TS in 19 cases $(14.8 \%)$. The frequency of registration TO $>0 \%$ and TS $<2.5 \mathrm{~ms} / \mathrm{RR}$ significantly dominated in the group with restrictive type of LV DD in comparison with group I (DD type of abnormal relaxation) and group II (DD of pseudonormal type), $\mathrm{p}<0.05$.

We have established a connection of HRT violation with left atrium volume change indicator- $r=-0.43(p=0.032)$, with left ventricular ejection fraction- $r=-0.49(p=0.03)$, with end-diastolic volume index $-r=0.51(p=0.02)$, with left ventricular mass index $-r=0.45(p$ $=0.04)$. We marked the correlation of TS with HRV indicators: SDNN $(r=0.41, p=0.02)$ and $\mathrm{LH} / \mathrm{HF}(\mathrm{r}=0.332, \mathrm{p}=0.02)$. The absolute values of TO significantly correlated with QTd $(\mathrm{r}=$ $0.349, \mathrm{p}=0.04)$.

Pathophysiologic ground for coronary heart disease is a discrepancy between myocardial oxygen demand and opportunities in meeting the needs in oxygen because of a limitation of acute or chronic coronary blood flow. Admittedly, the provision of myocardial blood through the coronary arteries occurs in the phase of diastole. That is why we suggest to consider diastolic dysfunction not only as an initial stage, but also as a pathological link in the "vicious circle" of the development of cardiac events in CHD patients.

Rates of diastole and its function undergo complex changes caused by the violation of diastolic relaxation in the background of acute or chronic ischemia, with increased stiffness of the myocardium at the site of postinfarction scar during the coronary heart disease. Necrosis of cardiomyocytes and activation of the cascade of biochemical reactions which occur in the postinfarction period, cause a significant change in mechanical properties of tissues. However, we should take into account different biomechanical properties of myocardium in the areas of chronic ischemia and so-called intact (healthy) myocardium. The specific effects are generated on their borders according to the type of stress concentration. They cause the further progression of degenerative and sclerotic changes, the formation of connective tissue in conditions of limited blood flow. In conditions of chronic ischemia, the changes occur not only in cardiomyocytes but also in cells of connective tissue skeleton. Increased rigidity of myocardium, change in the myocardium viscoelastic properties are the consequences of disorganization and reorganization of connective tissue skeleton of ischemic nature. Progressive hypertrophy and dilation of the heart are accompanied by a further violation of the diastolic left ventricular function, then it is accompanied by systolic dysfunction, they cause an increase in myocardial oxygen demand and a change in subendocardial blood flow, impaired myocardial bioenergetics.

The evaluation of the changes of myocardium electrophysiological properties seems for us to be an important aspect in the search for new diagnostic tests of severity of ischemic myocardial damage in patients with coronary heart disease. The validity of these approaches is based on the notion that electrophysiological alternation of cells and their membranes is associated with remodeling after an episode of ischemia or myocardial 
infarction and is involved in genesis of arrhythmia That is why we examined the relationship of electrical and structural myocardium remodeling with left ventricular diastolic function in CHD patients who had myocardial infarction history.

The analysis of ventricular late potentials and dispersion of QT interval allows to assess arrhythmogenic substrate in patients having CHD with left ventricular diastolic dysfunction. Persistent deterioration of left ventricular diastolic function during myocardium hibernating among CHD patients increases the manifestation of inhomogeneity of depolarization and repolarization processes.

The increase of the sympathetic influence has an effect on myocardium active relaxation and diastolic phase structure during the subsequent abuse of metabolic pathways accompanied by destabilization of connective tissue skeleton and secondary changes of contractile myocardium. Neurohumoral regulation violations caused by diastolic dysfunction in $\mathrm{CHD}$ patients are the same as when they are generated by heart failure. They are evident in increase of sympathetic activity, reduced parasympathetic and pressosensitive activity.

Even single ventricular extrasystole can be a trigger factor of fatally dangerous arrhythmias in conditions of chronic myocardial ischemia with arrhythmogenic substrate, which is the marked by ventricular late potential and QTd, when autonomic nervous system is activated according to the sympathetic type.

Complex analysis of parameters reflecting the functional conditions of myocardium and the interrelation of electrical and structure-geometrical myocardium remodeling is necessary to improve the diagnostics and the prognostication of risk degree among CHD patients in conditions of increasing of left ventricle diastolic dysfunction, given the multifactorial genesis of the problem of myocardial electrical instability

The use of integrated risk markers may contribute to the development of an integrated approach to risk stratification of SCD and arrhythmic complications prevention in CHD patients.

\section{Abbreviations}

ANS - autonomic nervous system

CHD - coronary heart disease

$\mathrm{CHF}$ - chronic heart failure

DD - diastolic dysfunction

DF - diastolic function

EDD - end-diastolic size

EDV - end-diastolic volume

ESD - end- systolic size

EHI - electrical heart instability

ESV - end- systolic volume 
EF - ejection fraction

HRT - heart rate turbulence

HRV - heart rate variability

IVRT - isovolumic relaxation time

LV - left ventricle

LVMI - left ventricular mass index

MI - myocardial infarction

SA - supraventricular arrhythmias

SCD - sudden cardiac death

SI - stroke index

$\Delta S$ - systolic shortening's fraction of the front-back size aortic ventricle

VA - ventricular arrhythmia

VLP - ventricular late potential

\section{References}

[1] Allender S., Scarborough P., Peto V., Rayner M., Leal J., Luengo-Fernandez R., Gray A. . European cardiovascular disease statistics 2008 / / Eur. Heart Network. -2008.- Vol. 4. - P. 76-78.

[2] Kannel W.B., Feinleib M. Natural history of angina pectoris in the Framingham study. Prognosis and survival // American Journal of Cardiology. - 1972.- Vol. 29.- P. 154-163.

[3] Pepine C.J., Abrams J., Marks R.G. et al. Characteristics of a contemporary population with angina pectoris: TIDES Investigators // Am. J. Cardiol. - 1994.- Vol. 74.- P. 226-231.

[4] Williams S.V., Fihn S.D., Gibbons R.J. Guidelines for the management of patients with chronic stable angina: diagnosis and risk stratification// Ann. Intern. Med. - 2001.Vol. 135.- P. 530-547.

[5] Oganov R.G., Maslennikova. G.Y. Demographic situation and cardiovascular diseases in Russia: ways to solve problems// Cardiovascular therapies and prevention. .2007.- №6. - P.7-14.

[6] Cowie M.R., Fox K.F., Wood D.A. et al. Hospitalization of patients with heart failure: a population-based study. / / Eur Heart J. - 2002.- Vol. 23.- P. 877-85.

[7] Cohn, J.N., Johnson G. Veterans Administration Cooperative Study Group. Heart failure with normal ejection fraction: V-HEFT Study // Circulation. - 1990.- Vol. 81.- P. 4853.

[8] O'Callaghan P.A., Camm A.J. Treatment of arrhythmias in heart failure // Europ. J. Heart Failure.- 1999.- Vol.1 (2).- P. 133-137. 
[9] Mareev V.Y. Heart failure and ventricular arrhythmias: the problem of treatment.// Cardiology.-1996.- № 12.- P.4-12.

[10] Cowie M.R., Mosterd A., Wood D.A. et al. The epidemiology of heart failure // Eur Heart J. - 1997. - Vol. 18.- P. 208-255.

[11] Devereux R.B. Left ventricular diastolic dysfunction: early diastolic relaxation and late diastolic compliance. J. Am. Coll. Cardiol 1989; 13: 337-339.

[12] Kapelko V.E. The evolution of concepts and metabolic basis of ischemic myocardial dysfunction.// Cardiology. - 2005. - № 9. - P. 55-61.

[13] Wainwirght C.L. Matrix metalloproteinases? Oxidative stress and the acute response to acute myocardial ischemia-reperfusion // Curr Opin Pharmacol. - 2004.- Vol. 4.P. $132-138$.

[14] Molkentin J.D., Lu J-R, Antos C.L. et al. A calcineurin-dependent transcriptional pathway for cardiac hypertrophy / / Cell.- 1998. - Vol. 93.- P. 215 - 228.

[15] Soto J., Beller G. Clinical Benefit of Noninvasive Viability Studies of Patients with Severe Ischemic Left Ventricular Dysfunction / / Clin. Cardiol. - 2001. - Vol. 24. - P. 428-434.

[16] Opie L.H. Newly identified ischemic syndromes and myocardial endogenous cytoprotection and their role in clinical cardiology in the past and future. // Medicography. - 1999. - № 21(2). - P. 65-73.

[17] Hohnloser S.H., Franck P., Klingenheben T. et al. Open infarct artery, late potentials, and other prognostic factors in patients after acute myocardial infarction in the thrombolytic era: a prospective trial / / Circulation. - 1994.- Vol. 90.- P. 1747-1756.

[18] Goldstein S., Brooks M. M., Ledingham R. et al. Association between ease of suppression of ventricular arrhythmia and survival/ / Circulation. - 1995.- Vol. 91.P. 7979 - 7983.

[19] Petersen S., Peto V., Rayner M. et al. European cardiovascular disease statistics // Eur. Heart J. - 2005. - Vol. 21. - P. 1261-1272.

[20] Kramer J.B., Saffitz J.E., Witkowsky F.V., Corr P.B. Intramural reentry as a mechanism of ventriculartachycardia during evolving canine myocardial infarction.// Circ. Res.- 1985 - Vol. 56 - P. 736-754.

[21] Tatarchenko E.P., Pozdnyakova N.V., Morozova O.E. Late potentials of the heart: clinical and electrophysiological evaluation. Penza: Elma.- 2000. - P. 144 .

[22] Maisch B. Ventricular remodeling // Cardiology.- 1996.- Vol. 8(7).- P. 2-10.

[23] Bigger J., Fleiss J.L., Steiman R.C. Correlation among time and freguency domain measures of heart period variability two week after acute myocardial infarction. Am. J. Cardiol 1992; 69: 891 - 898.

[24] Tatarchenko E.P., Pozdnyakova N.V., Morozova O.E., Shevyryov V.A. Prognosis of patients with coronary heart disease (clinical and instrumental aspects). Penza: Elma.- 2002. - P. 206.

[25] Schmidt G., Malik M., Barthel P. et al. Heart-rate turbulence after ventricular premature beats as a predictor of mortality after acute myocardial infarction / / Lancet.- 1999.Vol.353.- P.1390-1396. 
[26] Ghuran A., Reid F., La Rovere M.T. et al. Heart rate turbulence-based predictors of fatal and nonfatal cardiac arrest (The Autonomic Tone and Reflexes After Myocardial Infarction substudy) // Am. J. Cardiol.- 2002.- Vol.89.- P.184-190

[27] Bauer A., Barthel P., Schneider R., Schmidt G. Dynamics of heart rate turbulence // Circulation.- 2001.- Vol.104, Supplement II-339.- P.1622. 


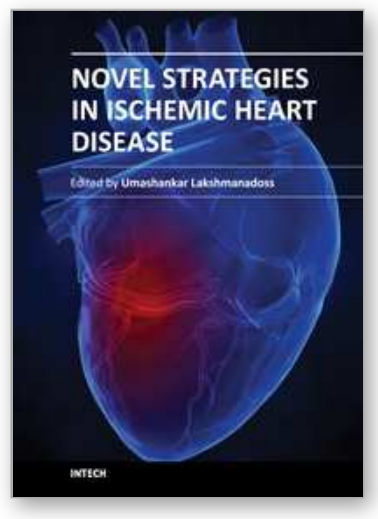

\author{
Novel Strategies in Ischemic Heart Disease \\ Edited by Dr. Umashankar Lakshmanadoss
}

ISBN 978-953-51-0184-0

Hard cover, 450 pages

Publisher InTech

Published online 29, February, 2012

Published in print edition February, 2012

The first edition of this book will provide a comprehensive overview of ischemic heart disease, including epidemiology, risk factors, pathogenesis, clinical presentation, diagnostic tests, differential diagnosis, treatment, complications and prognosis. Also discussed are current treatment options, protocols and diagnostic procedures, as well as the latest advances in the field. The book will serve as a cutting-edge point of reference for the basic or clinical researcher, and any clinician involved in the diagnosis and management of ischemic heart disease. This book is essentially designed to fill the vital gap existing between these practices, to provide a textbook that is substantial and readable, compact and reasonably comprehensive, and to provide an excellent blend of "basics to bedside and beyond" in the field of ischemic heart disease. The book also covers the future novel treatment strategies, focusing on the basic scientific and clinical aspects of the diagnosis and management of ischemic heart disease.

\title{
How to reference
}

In order to correctly reference this scholarly work, feel free to copy and paste the following:

E.P. Tatarchenko, N.V. Pozdnyakova, O.E. Morozova and E.A. Petrushin (2012). Electrical Heart Instability Evaluation in Conditions of Diastolic Heart Failure Suffered by Coronary Heart Disease Patients, Novel Strategies in Ischemic Heart Disease, Dr. Umashankar Lakshmanadoss (Ed.), ISBN: 978-953-51-0184-0, InTech, Available from: http://www.intechopen.com/books/novel-strategies-in-ischemic-heartdisease/electrical-heart-instability-evaluation-in-conditions-of-diastolic-heart-failure-suffered-by-coronar

\section{INTECH}

open science | open minds

\section{InTech Europe}

University Campus STeP Ri

Slavka Krautzeka 83/A

51000 Rijeka, Croatia

Phone: +385 (51) 770447

Fax: +385 (51) 686166

www.intechopen.com

\section{InTech China}

Unit 405, Office Block, Hotel Equatorial Shanghai

No.65, Yan An Road (West), Shanghai, 200040, China

中国上海市延安西路65号上海国际贵都大饭店办公楼405单元

Phone: +86-21-62489820

Fax: +86-21-62489821 
(C) 2012 The Author(s). Licensee IntechOpen. This is an open access article distributed under the terms of the Creative Commons Attribution 3.0 License, which permits unrestricted use, distribution, and reproduction in any medium, provided the original work is properly cited. 